Textures and Microstructures, 1989, Vol. 10, pp. 101-116

Reprints available directly from the publisher

Photocopying permitted by license only

(C) 1989 Gordon and Breach Science Publishers Inc.

Printed in the United Kingdom

\title{
An Improvement of Vector Method by Allocation of Intensities Based on the Crystallographic Symmetry
}

\author{
R. SHIMIZU, K. OHTA and J. HARASE \\ R\&D Laboratories III, Nippon Steel Corporation, Edamitu, Yawata-Higashiku, \\ Kitakyusyu 805, Japan
}

(Received 20 June 1988; in final form 6 August 1988)

An investigation has been carried out utilizing model and experimental pole figures made by X-ray technique in order to examine the use of the vector method as a means of the texture analysis. The main findings are as follows:

- From crystal symmetry considerations positions and magnitudes of peaks along the $\zeta$ angle can be predicted. There are discrepancies in these intensity peaks and in some cases the peaks are missing altogether.

- This problem was solved by the allocation of intensities such that equal intensities are obtained at the crystallographic symmetry positions.

- Even a slightly mismatched combination of the reflection and transmission pole figures caused an increase in residual vector $(R)$ resulting in the failure of the analysis for the minor textural component.

KEY WORDS: ODF analysis, vector method, crystal symmetry, $\mathrm{Fe}-3 \% \mathrm{Si}$.

\section{INTRODUCTION}

The Vector method (VM), the Harmonic method (HM) and the WIMV method are used to invert pole figure. Several investigations have been done on the comparison of these methods (e.g. Kitagawa et al., 1982; Pospiech et al., 1984, Matsuo et al., 1985, Esling et al., 1987). 
The HM is the most commonly used pole figure inversion technique. The present authors, however, have used the VM for about 2000 pole figure inversions during research and development of the engineering materials. In VM analysis, it is possible to invert from only one complete (100) pole figure. However, if accuracy is not of prime importance, it is also possible to invert from an incomplete (110) pole figure $\left(\alpha_{\max } \geqq 75^{\circ}\right)$.

In the inversion of pole figures, the $\mathrm{VM}$ is very efficient during preprocessing stage compared with that of the HM, however, the time required for the calculation in the VM is considerably longer than in the HM. The need for larger computers to handle the VM has restricted its use, whereas minicomputers can be used in HM analysis. As an illustration of this for the case of the VM, using an iteration of 150 , the time required for the calculation is about $12 \mathrm{hr}$, $1 \mathrm{hr}$ and $6 \mathrm{~min}$ for analysis on IBM 4341, on IBM 3083 and on IBM 3090 , respectively. In this work the analysis is done on IBM 3090 , the primary rate determining factor in the texture analysis by the $\mathrm{VM}$, becomes the collection of the required X-ray data. The VM is therefore efficient in the time required to measure and analyse of the pole figure, however, the accuracy of the VM compared with other methods remains uncertain.

During preliminary experiments to compare the VM and the HM, the present authors found that the intensities analyzed by the VM and the HM are not always in good agreement. Also for the VM, the intensities of some textures which from crystallographic symmetry considerations should be equal, were found to be dissimilar within the same values (Kitagawa et al., 1982; Shimizu et al., 1984)). In order to evaluate the characteristics of the VM and to improve the method to make it a practical means of texture analysis, investigations have been done utilizing both model and experimental pole figures made by the $\mathrm{X}$-ray measurements.

\section{INVESTIGATION OF THE VM UTILIZING MODEL POLE FIGURES}

The model pole figures utilized are shown in Table 1 . They consist of 8 basic orientations and combinations of these basic orientations. $A$ and $B$ in Table 1 means that either of them has - sign in any of 
Table 1 Model (100) pole figures used for the investigation of the VM

\begin{tabular}{|c|c|c|c|c|c|c|c|c|c|c|c|c|c|c|}
\hline \multirow{4}{*}{$\begin{array}{l}\text { Basic } \\
\text { Orienta- } \\
\text { Combi-tions } \\
\text { nations }\end{array}$} & \multirow{3}{*}{$\frac{1}{\frac{1}{\{001\}<011>}}$} & \multicolumn{2}{|c|}{ l } & \multicolumn{2}{|c|}{ 而 } & \multicolumn{2}{|c|}{ IV } & \multicolumn{2}{|c|}{ V } & n & \multicolumn{2}{|c|}{ III } & \multicolumn{2}{|c|}{ III } \\
\hline & & $\multicolumn{2}{|c|}{112}<011>$ & $\multicolumn{2}{|c|}{111}<112>$ & \multicolumn{2}{|c|}{$\frac{111}{\mid 111<01 D}$} & \multicolumn{2}{|c|}{$\frac{1}{\{133\}<011\rangle}$} & \multirow{2}{*}{ 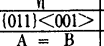 } & $\multicolumn{2}{|c|}{012}<001>$ & $\multicolumn{2}{|c|}{134}<111>$ \\
\hline & & $\mathrm{A}$ & $\mathrm{B}$ & $\mathrm{A}$ & $\mathrm{B}$ & $\mathrm{A}$ & $\mathrm{B}$ & $\mathrm{A}$ & $\mathrm{B}$ & & $\mathrm{A}$ & $B$ & $\mathrm{~A}$ & $\mathrm{~B}$ \\
\hline & 8 & D & 4 & $\Delta$ & $\bar{\nabla}$ & $\Delta$ & 4 & $\Delta$ & $D$ & पा & 므 & $\square$ & $\nabla$ & $\nabla$ \\
\hline $\mathrm{A}$ & & 0 & & 0 & & 0 & & 0 & & & 0 & & 0 & \\
\hline B & & & 0 & & 0 & & 0 & & 0 & & & $\mathrm{O}$ & & 0 \\
\hline $\bar{A}+B$ & 0 & & 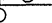 & & & & & & & $\mathrm{O}$ & & 5 & & \\
\hline $1+\pi$ & 0 & & & & & & & & & & & & & \\
\hline+11 & & & 5 & & & & & & & & & & & \\
\hline+7 & & & & & 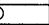 & & & & & & & & & \\
\hline+11 & & & & & 5 & & & & & 0 & & & & \\
\hline+1 & 0 & & 5 & & & & & & & & & & & \\
\hline+11 & 0 & & & & & & & & & 0 & & & & \\
\hline+1 & & & 5 & & & & & & & & & & & \\
\hline $1+11$ & & & 5 & & & & & & & 0 & & & & \\
\hline$\sqrt{+11}$ & & & & & & & & & & 0 & & & & \\
\hline $1+m+1$ & 0 & & & & 5 & & & & & & & & & \\
\hline $1+1+\sqrt{+11}$ & 0 & & 5 & & & & & & & 0 & & & & \\
\hline $\begin{array}{l}1+11+11+1+1 \\
+11+11+11\end{array}$ & 0 & & 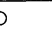 & & 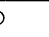 & & & & & 0 & & ) & & \\
\hline
\end{tabular}

*1 Odenotes orientations used in tho investigation.

*2 $A$ and $B$ in the Table means that either of them has a negative sign in any of the indicies $u, v, w$ of ihe direction $\langle u v w\rangle$. The mutual orientation relationship is shown in the etch pit.

the indices $u, v, w$, of the $\langle u v w\rangle$ direction and the mutual orientation relationship is shown in the shape of etch pit.

Two examples of (100) model pole figures are shown in Figure 1. In the preparation of the model pole figure from $(h k l)[u v w]$, the intensity distribution around exact $(\alpha, \beta)$ was given a Gaussian distribution. Here angle $\alpha$ denotes azimuth angle and $\beta$ denotes rotation angle from the rolling direction (RD). A clockwise rotation is assumed to be positive. The intensity distribution is given at each 5 degrees from both $\alpha$ and $\beta$, and the spread of the intensity distribution is about 2 degrees for the half width.

Among the eight basic orientations, orientations 2, 3, 4, and 7 have been selected and model pole figures composed of these

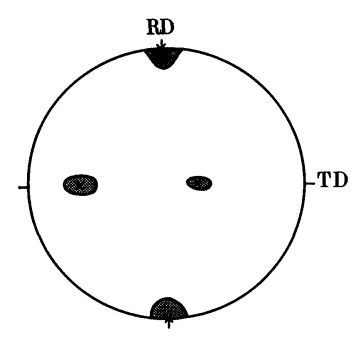

(a) $\{012\}<001\rangle$

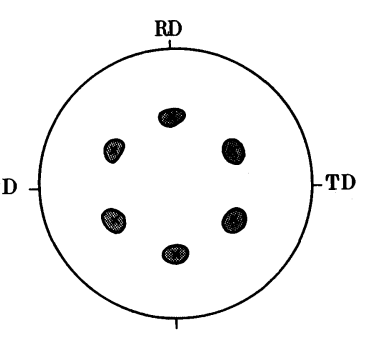

(b) $\{111\}<112\rangle$

Figure 1 Examples of (100) model pole figures. 


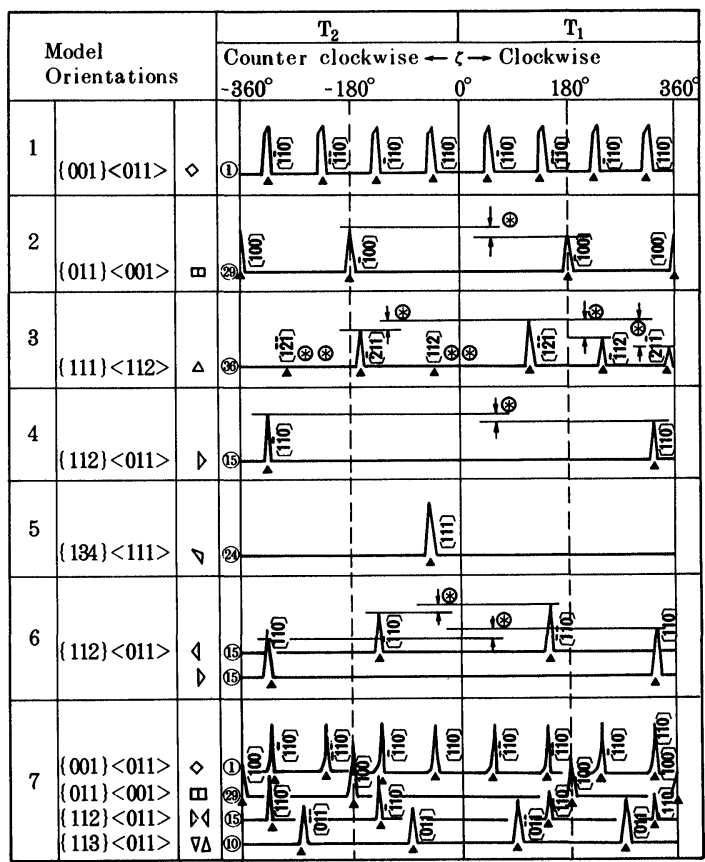

Figure 2 Inversion results of model pole figures by the vector method. Digits in the circle denote corresponding Box No. $\Delta$ indicates the position where the intensity peak should appear. ${ }^{*}$ indicates that a slight discrepancy in the intensity value exists. ** indicates the position where no peak intensity appears in a position where an intensity peak is expected.

orientations have been made. The mark $\bigcirc$ in Table 1 indicates the specific orientations selected for this purpose. Some of the inversion results obtained by the VM are shown in Figure 2. In the VM, the orientation of the given texture is represented by the Box number (Box No) and angle $\zeta$. The Box No is a function of $\psi$ and $\omega$. The figures in the circle denote the Box No and the intensity distributions are shown schematically as a function of $\zeta$ in regions $T_{1}$ and $T_{2}$ defined in the VM. $\zeta$ value $0^{\circ}$ to $+360^{\circ}$ and $0^{\circ}$ to $-360^{\circ}$ corresponds to $T_{1}$ and $T_{2}$ region respectively. Here the equivalent orientation group is shown schematically in Figure 3. It is defined as the orientation group in which the intensity peak appears periodically at specific intervals at $\zeta$ values corresponding to the crystallographic symmetry. 


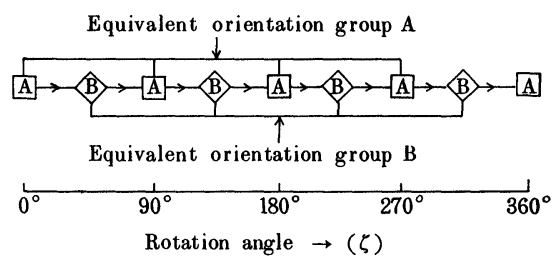

Figure 3 Schematic illustration of the definition of the equivalent orientation group. This is an example of the 4 fold symmetry.

As shown in Figure 2, the model orientation has its specific intensity peak position spaced periodically at the specific $\zeta$ angle according to its crystallographic symmetry. For example, $\{001\}\langle 011\rangle$ (model No1) has four fold symmetry and its $\zeta$ angle for the peak value should be $-315^{\circ},-225^{\circ},-135^{\circ},-45^{\circ}, 45^{\circ}, 135^{\circ}$, $225^{\circ}, 315^{\circ}$. The mark $\Delta$ in Figure 2 denotes the position where the intensity peak of the direction [uvw] should appear. $\circledast$ and $\circledast \circledast$ denote that the analyzed results are different from expected, namely, $\circledast$ indicates a discrepancy in the intensity level and $\circledast \circledast$ indicates that no intensity peaks appear in the position where they are expected from symmetry considerations.

In the model orientation 2 , the peak height is smaller in region $T_{1}$ compared to region $T_{2}$.

In the model orientation 3 , the three intensity peaks in $T_{1}$ are not the same magnitude and they are different from the single intensity peak in $T_{2}$. In the $T_{2}$ region, two of the expected intensity peaks are missing completely.

In the model orientation 4, 6 and 7 the magnitudes of intensity peaks in $T_{1}$ and $T_{2}$ are different. In model orientations 5 , only the expected peak at [111] is observed. From these observations, it is concluded that the VM method is not correct in the allocation of intensities when the model pole figures are used.

\section{INVESTIGATION UTILIZING EXPERIMENTAL POLE FIGURES}

Figure 4 shows experimental pole figure utilized for the investigation on the VM. It was made from a primary recrystallized $\mathrm{Fe}-3 \% \mathrm{Si}$. The typical orientations are shown with the marking of 


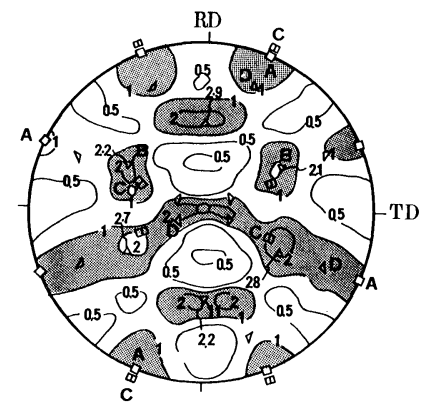

$A \diamond \diamond:\{001\}\langle 025\rangle, C \otimes \infty:\{011\}\langle 227\rangle$

$\mathrm{B} \nabla \Delta:\{111\}<112\rangle, \mathrm{D} \nabla \Delta \Delta:\{114\} \mathrm{RD} 22.5^{\circ}<011>$

Figure 4 A complete (100) pole figure made from the X-ray measurement of a primary recrystallized $\mathrm{Fe}-3 \% \mathrm{Si}$, polycrystalline material.

A, B, C, D. An inversion of this pole figure by the VM is shown in Figure 5. The marking A, B, C, D are the same as in Figure 4. The intensity distributions of $\mathrm{A}, \mathrm{B}, \mathrm{C}, \mathrm{D}$ along the $\zeta$ are shown in Figure 6. Figure 6 shows the discrepancies in the analysis of the results at equivalent orientations. The discrepancies are recognized within $T_{1}$ or $T_{2}$, or between $T_{1}$ and $T_{2}$. The discrepancy is especially large in the orientation $A$ with 4 fold symmetry and B with 3 fold symmetry.

Figure 7 shows the intensity of the inverse pole figure made from the X-ray measurements plotted against that obtained by the VM obtained by averaging the intensity within the same Box. In this case the residual vector $\mathrm{R}$ is less than $10 \%$ and the pole figure inversion inverts even the minor texture component. The (111), (112), (001), (011) orientation in the inverse pole figure corresponds to Box No 36, 15, 1 and 29 in the VM respectively. Good agreement was obtained between intensities obtained directly from $\mathrm{X}$-ray measurement and the average VM intensities in the corresponding Box No.

\section{ALLOCATION OF THE INTENSITY USING CRYSTALLOGRAPHIC SYMMETRY CONSIDERATIONS}

The main problem with the VM analysis concerns the intensity distribution along the $\zeta$ angle. It appears necessary to adjust the 


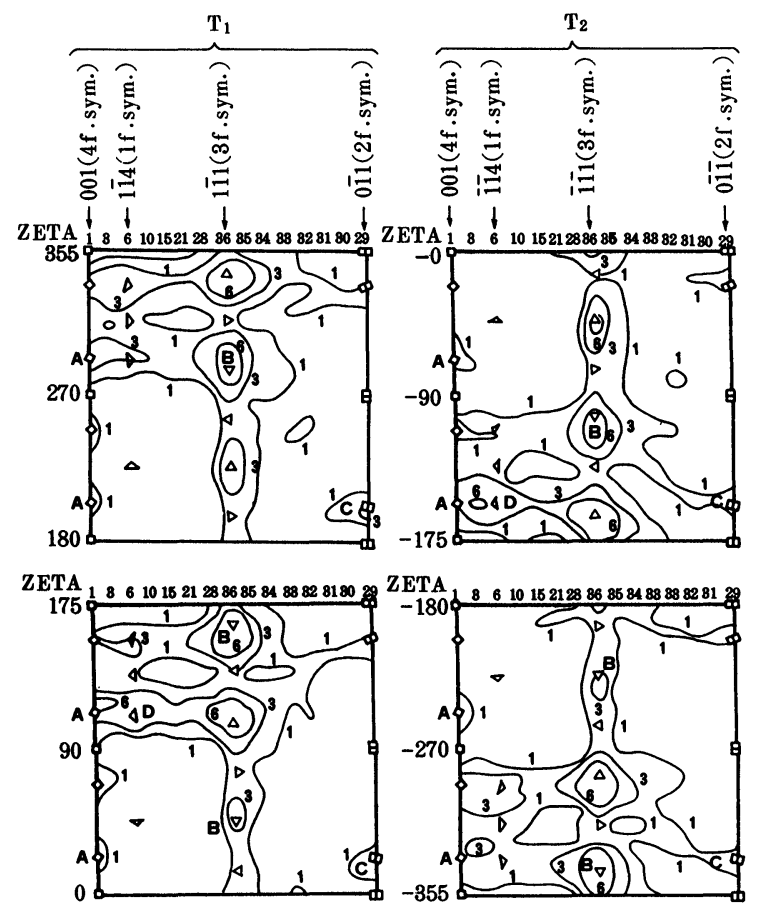

Figure 5 VM-Inversion results from the experimental pole figure shown in Figure 4.

intensity distribution along the $\zeta$ angle by taking into account the periodicity of the intensity peak due to the crystal symmetry. This can be done as follows:

(1) The periodicity of the intensity peaks depends on the crystal symmetry of the given orientations. For a 4 fold symmetry, the intensity peak is present at each $\zeta=90^{\circ}$ period. For 3 and 2 fold symmetry, the intensity peak is present at each $\zeta=120^{\circ}$ and $180^{\circ}$ period respectively. In the case of one fold symmetry, it changes depending on the representation mode. Here the term representation mode is the mode representing the final results analyzed by the VM and consists of symmetrical and asymmetrical modes. The symmetrical mode is shown by analyzing results between $0^{\circ}$ to $180^{\circ}$ in $\zeta$ angle. The asymmetrical mode is shown by analyzing the 


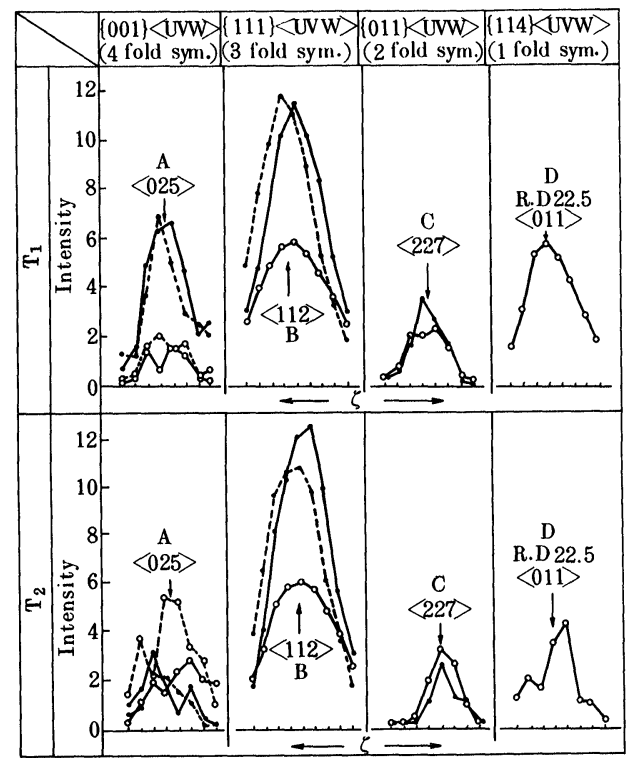

Figure 6 A schematic representation of the intensity distribution around the $\zeta$ angle demonstrating a discrepancy in the magnitude of the intensity peak value among the equivalent orientation groups.

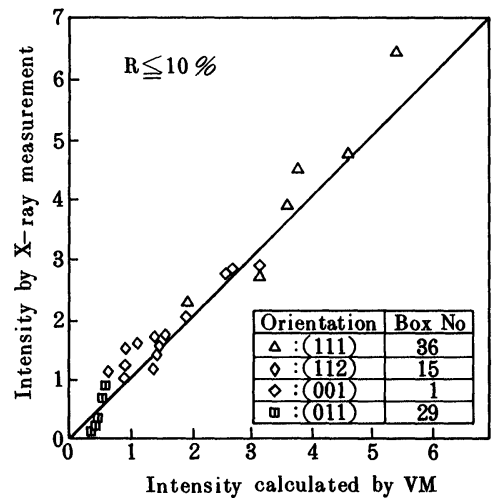

Figure 7 The relationship between the inverse pole figures obtained directly from the X-ray measurement and that calculated from the results by VM. 


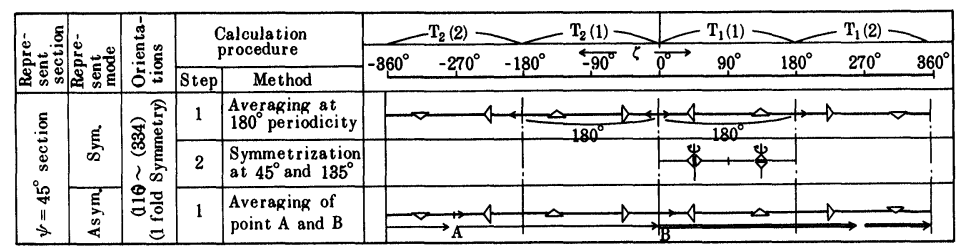

Figure 8 Schematic illustration of the calculation procedure and its representation method, for the allocation of the intensities of the equivalent orientation groups for the case of one fold symmetry.

results between $0^{\circ}$ to $360^{\circ}$ in $\zeta$ angle. In both cases they are shown in the $T_{1}$ region. Using this terminology a direct comparison with the harmonic method can be made (Nagashima et al., 1981).

(2) The method of the allocation of the intensities in the case of one fold symmetry is shown in Figure 8. In this figure, the left side column indicates the section of representation which is the same as the HM, namely, $\psi=45^{\circ}$ section. The representation mode of the next column is as explained above. Orientations chosen as one fold symmetry are (116)-(334). The calculation procedure is as follows.

\section{(i) Symmetrical mode}

Step 1: The intensity is averaged at a periodicity of $180^{\circ} . I\left(\zeta_{i}\right)=$ $\left[I\left(\zeta_{i}\right)+I\left(\zeta_{i}+180^{\circ}\right)\right] / 2$ for $\zeta=0^{\circ}-180^{\circ}, \zeta=0^{\circ}--180^{\circ}$ in the region of $T_{1}$ and $T_{2}$ respectively. Here $I\left(\zeta_{i}\right)$ denotes the intensity at $\zeta=\zeta_{i}$. Then $I\left(\zeta_{i \text { ave }}\right)=\left\{I\left(\zeta_{i}\right)+I\left(-\zeta_{i}\right)\right\} / 2$ is calculated and they are displayed in the region of $T_{1}(1)$ for $\zeta=0^{\circ}-180^{\circ}$.

Step 2: The result obtained at step 1 is processed so that the intensity distribution between $\zeta=0^{\circ}-90^{\circ}$ becomes symmetric around $\zeta=45^{\circ}$, and the intensity distribution between $90^{\circ}-180^{\circ}$ becomes symmetric around $\zeta=135^{\circ}$ in the following manner,

$$
\begin{gathered}
I\left(45^{\circ}-\Theta\right)=I\left(45^{\circ}+\Theta^{\circ}\right)=\left[I\left(45^{\circ}-\Theta^{\circ}\right)+I\left(45^{\circ}+\Theta^{\circ}\right)\right] / 2 \\
I\left(135^{\circ}-\Theta^{\circ}\right)=I\left(135^{\circ}+\Theta^{\circ}\right)=\left[I\left(135^{\circ}-\Theta^{\circ}\right)+I\left(135^{\circ}+\Theta^{\circ}\right)\right] / 2
\end{gathered}
$$

(ii) Asymmetrical mode. The average intensity of point $\mathrm{A}(\zeta=$ $\left.-270^{\circ}\right)$ and point $B\left(\zeta=0^{\circ}\right)$ is assigned the intensity of point $B$ and continuing the same calculation by increasing the $\zeta$ value, the new intensity at $\zeta^{0}$ can be expressed as follows,

$$
I\left(\xi^{0}\right)=\left[I\left(\zeta^{0}\right)+I\left(-270^{\circ}+\zeta^{0}\right)\right] / 2
$$




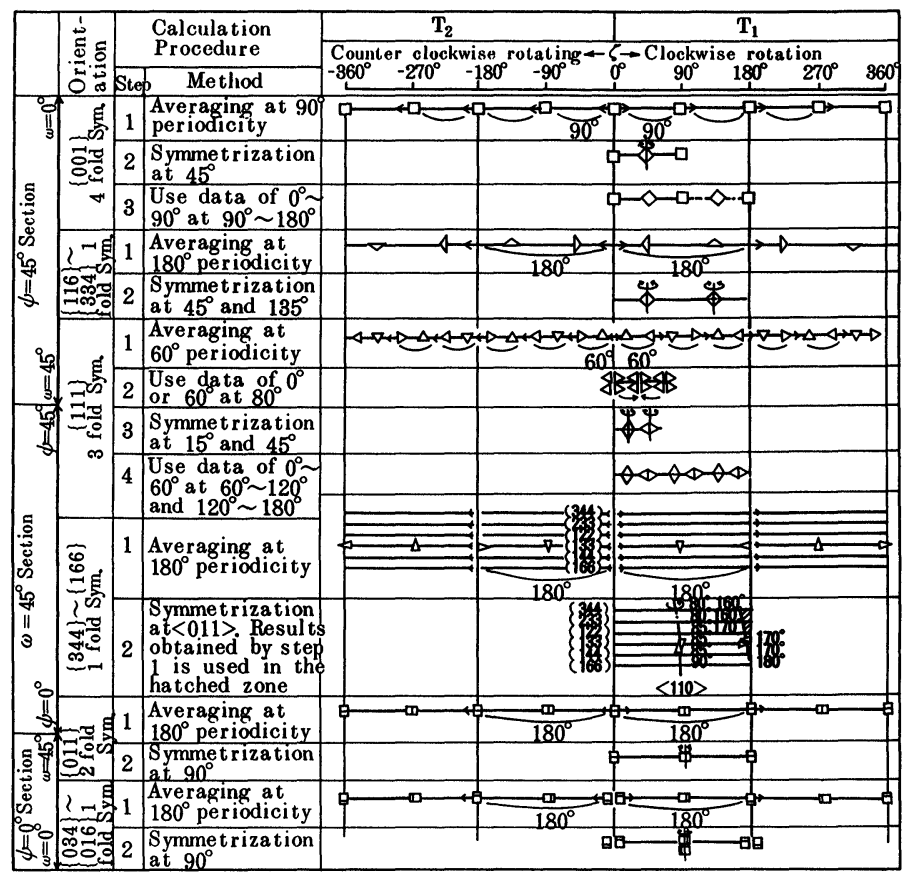

Figure 9 Schematic illustration of the calculation procedure and its representation method for the allocation of the intensities of equivalent orientation groups (symmetrical mode).

Here point A jumps to $\zeta=-360^{\circ}$ when point A comes to the position where $\zeta=0^{\circ}$. By this calculation, all the intensity distributions are shown in the $T_{1}$ region between $\zeta=0^{\circ}$ and $360^{\circ}$. So far the case of one fold symmetry at the $\psi=45^{\circ}$ section only has been explained. The case of the $\omega=45^{\circ}$ section is shown in Figures 9 and 10.

(3) The whole scheme of the allocation of the intensities along the $\zeta$ angle is shown in Figures 9 and 10. One fold symmetry is the most complicated case. In the case of the symmetrical mode shown in Figure 9 , the $\zeta$ value for the center of the symmetry is slightly different according to the orientations in the one fold symmetry at the $\omega=45^{\circ}$ section.

In the case of the asymmetrical mode shown Figure 10, the starting point $\mathrm{A}$ in region $T_{2}$ is different according to orientation 


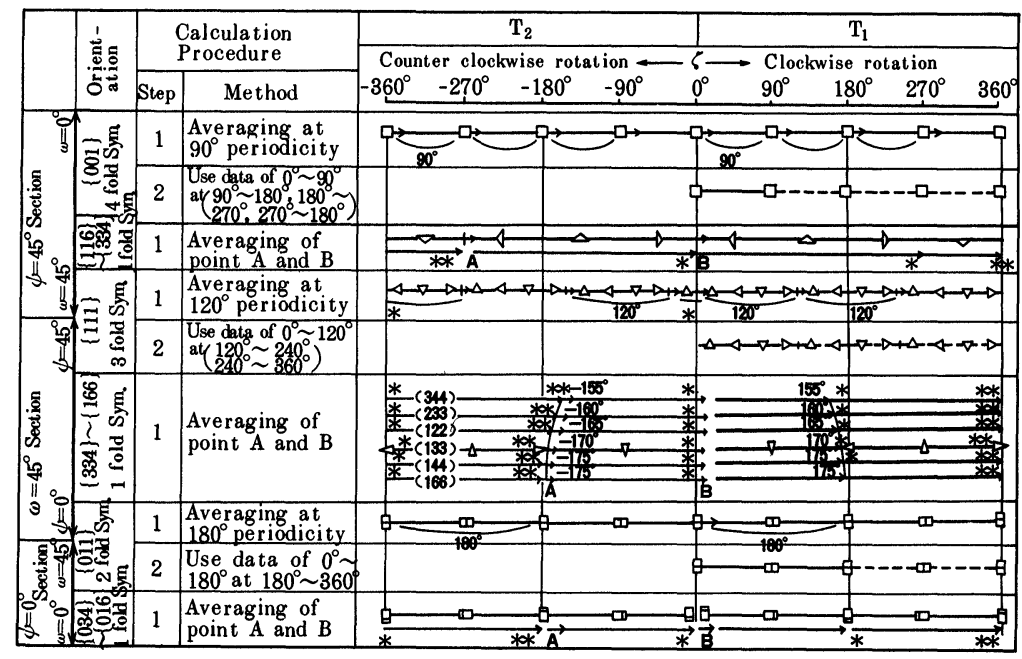

Figure 10 Schematic illustration of the calculation procedure and its representation method for the allocation of intensities of the equivalent orientation groups (asymmetrical mode).

when dealing with the $\omega=45^{\circ}$ section. Markings * and ${ }^{* *}$ in Figure 10 denote that the positions of similar marks have equivalent intensities. The results of the analysis by the VM taking into account an allocation of the intensities as described above are shown in Figure 11 and Figure 12 for symmetrical and asymmetrical modes respectively. The pole figure utilized was the one shown in Figure 4. A comparison of the analyzed results obtained by HM and those obtained by the VM after the allocation of the intensities is shown in Figure 13. The section of $\phi=45^{\circ}$ in the HM corresponds to the shaded parts of the results obtained by VM (Nagashima et al., 1981). Good agreement is obtained.

\section{THE RELATIONSHIP BETWEEN THE RESIDUAL VECTOR R AND THE CONSISTENCY OF THE INTENSITIES IN THE INPUT POLE FIGURE DATA}

It is known that the number of iterations $I_{N}$ used in the VM is closely associated with the values of residual vector $R$ and $Y \max$ (e.g. Shimizu et al., 1984). 


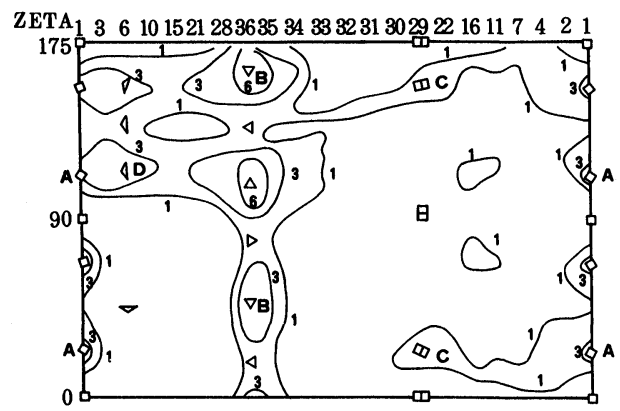

Figure 11 Inversion results of pole figure by VM using an allocation of intensities of equivalent orientation groups (symmetrical mode).

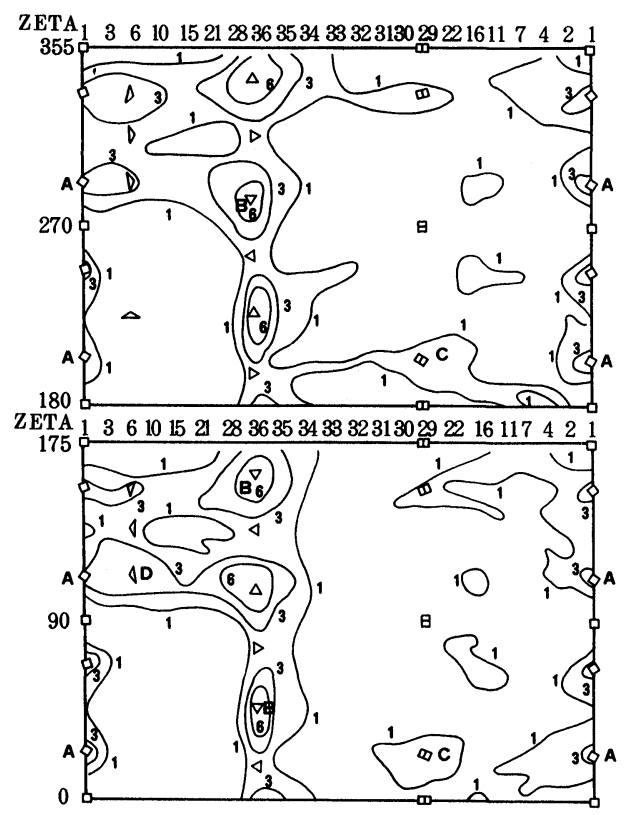

Figure 12 Inversion results of pole figure by the VM using an allocation of intensities of equivalent orientation groups (asymmetrical mode). 

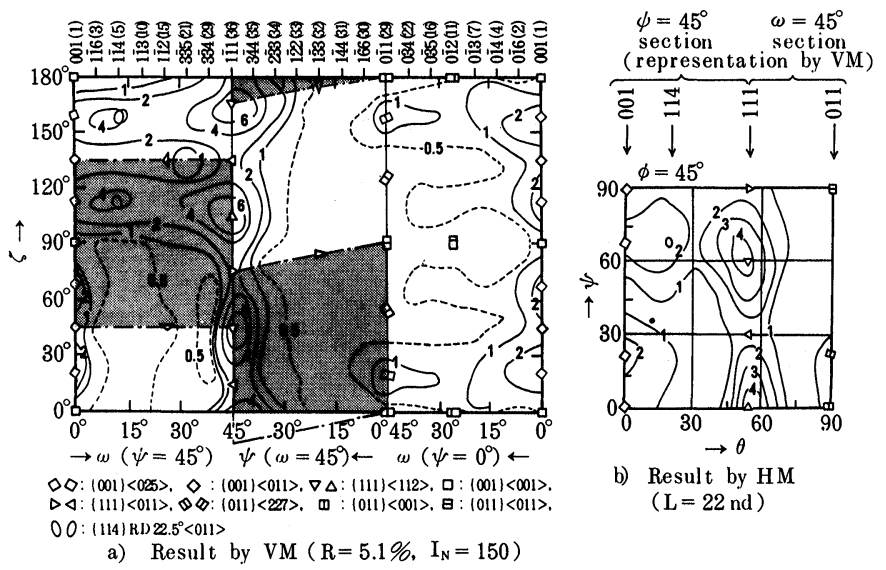

Figure 13 Comparison of the inversion results by VM and HM.

In general, $R$ decreases and $Y$ max increases with increasing $I_{N}$, and when $R$ is less than $10 \%, Y$ max becomes nearly $100 \%$. In this situation, the minor texture components are detected and the analyzed results by the VM become useful for the engineering purpose. In some cases this $R$ value doesn't decrease in spite of the increase in $I_{N}$. This is sometimes associated with the combination of the reflection and transmission pole figure in order to make the complete pole figure. It is known that a given single orientation gives 3 points $(\alpha, \beta)$ in the (100) pole figure and if their intensities are not consistent, the $R$ value doesn't decrease in spite of the increase of $I_{N}$.

An example is shown in Figure 14, where the pole intensity distribution is shown as a function of angle $\beta$ for each angle $\alpha$ instead of the pole figure more usually presented. The main texture component is (110)[001] and it contains cube texture as a minor component. In the as measured condition (as in 'Before correction'), the intensity of the cube component at $\alpha=0^{\circ}$ is slightly stronger compared to the component at $\alpha=90^{\circ}$. The intensity distribution for $\alpha=0^{\circ}-25^{\circ}$ is taken only from the reflection pole figure measurement and $\alpha=75^{\circ}-90^{\circ}$ is taken only from the transmission pole figure measurement. The corrected value ('After 


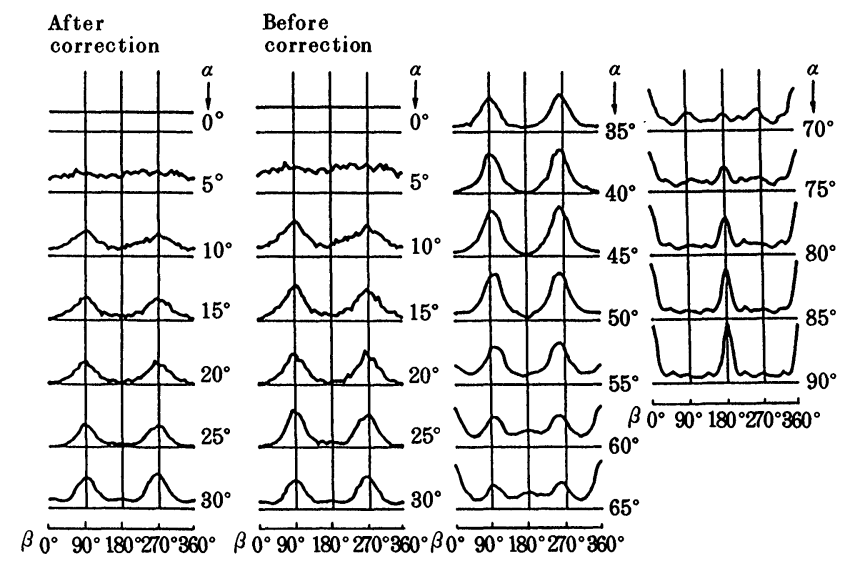

Figure 14 Intensity distribution obtained by reflection and transmission $x$-ray measurements. $\alpha=0^{\circ}-25^{\circ}$ range was determined from the reflection $\mathrm{x}$-ray measurements in this case. 'Before correction' denotes the results from the reflection $\mathrm{x}$-ray measurement without any correction and 'After correction' denotes that the intensities were slightly reduced to be consistent with the intensities obtained by the transmission $\mathrm{x}$-ray measurement.

correction') for $\alpha=0^{\circ}-25^{\circ}$ is shown in the left hand side of the original results. The corrected intensities are slightly reduced. A new pole figure was made utilizing this value and the original value for $\alpha=30^{\circ}-90^{\circ}$. $Y \max$ and $R$ values are shown in Figure 15 as a function of $I_{N}$ using the original and the corrected pole figures. It can be seen from Figure 15 that even the very small discrepancy in the intensity, which is very difficult to recognize from the pole figure, gives a very large effect when analyzed by the Vector method. It is therefore most important to have a consistent complete pole figure if the vector method is to be applied to the analysis of the data (Schaeben, 1984).

\section{CONCLUSIONS}

- The main problem with the Vector method concerns the intensity distribution along the $\zeta$ angle.

- Using modified peak intensity allocations, determined by crystal symmetry considerations, improved inversion results 


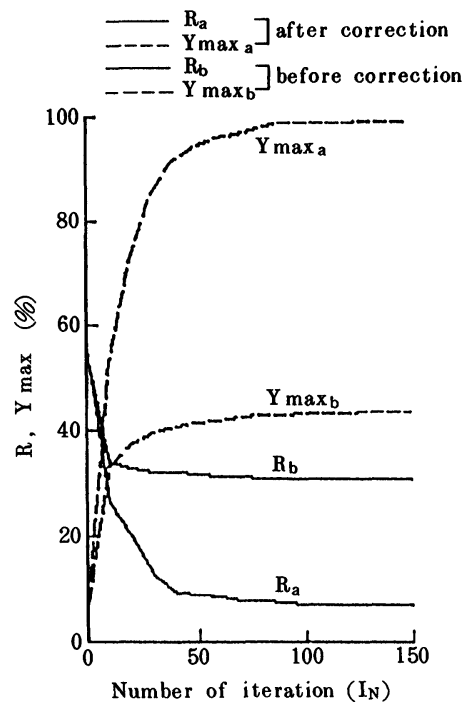

Figure 15 The relationship between the number of iterations and the values of $R$ and $Y \max$ for the two different pole figure inputs shown in Figure 14.

have been obtained using the Vector method analysis. The modified technique has been successfully used as a means of texture analysis of silicon irons and in the research and development of other engineering materials.

- Good agreement has been obtained, using the improved VM and the $\mathrm{HM}$, in the analysis of experimental pole figures of polycrystalline specimens.

- The inversion of the pole figure by the VM is however very sensitive to the consistency of the pole figure. Very well prepared pole figures with a residual vector $R$ less than $10 \%$ are required if the minor textured components are to be determined correctly by a vector method inversion.

\section{Acknowledgment}

In the present investigation, the authors used a Japanese computer program for the vector method analysis named as $\sigma-N$. This program was introduced to Japan by former Prof. Nagashima through the courtesy of the Profs. Ruer and Baro at the 
ICOTOM 6 in Japan. The authors also thanks Prof. Nagashima for his useful advice on the use of this program.

We would also like to express sincere thanks for Profs. Ruer, Baro and Nagashima emphasizing that the vector method has proved a very useful tool in the research and development of engineering materials within the Nippon Steel Corporation. We would finally like to express sincere thanks for Dr. R. Davidson at Harwell in the UK for his critical reading and revising of this manuscript.

\section{References}

Esling, C., Bunge, H. J., Philippe, M. J. and Muller, J. (1987). Theoretical Methods of Texture Analysis. Ed.; H. J. Bunge. DGM. Oberursel.

Kitagawa, H., Morimoto, K. and Iwasaki, Y. (1982). Tetsu to Hagané, 68, S1351.

Matsuo, M., Kawasaki, K. and Sugai, T. (1985). Tetsu to Hagané, 71, S1351.

Nagashima, S., Tanaka, K. and Nishikawa, S. (1981). Tetsu to Hagané, 67, S1198.

Pospiech, J., Romanska, M. and Lücke, K. (1984). Procc. 7th Inter. Conf. on Tex. Mat., 823.

Shaeben, H. (1984). Procc. 7th Inter. Conf. on Tex. Mat., 833.

Shimizu, R. and Ohta, K. (1984). Tetsu to Hagané, 70, S565. 